\title{
Co-release of GABA does not occur at glycinergic synapses onto lumbar motoneurons in juvenile mice
}

\author{
G. S. Bhumbra ${ }^{\ddagger}$, N. J. Moore ${ }^{\ddagger}$, M. Moroni ${ }^{\dagger}$ and M. Beato* \\ Department of Neuroscience, Physiology and Pharmacology, University College London, London, UK
}

Edited by:

Enrico Cherubini, International School for Advanced Studies, Italy

Reviewed by:

Annalisa Scimemi, National Institutes of Health, USA

Jerzy W. Mozrzymas, Wroclaw

Medical University, Poland

*Correspondence:

M. Beato, Department of

Neuroscience, Physiology and

Pharmacology, University College

London, Gower Street, London WCE1

6BT, UK

e-mail:m.beato@ucl.ac.uk

${ }^{\dagger}$ Present address:

M. Moroni, Max-Delbrück-Centrum

für Molekulare Medizin, Berlin-Buch,

Robert-Rössle-Street 10, 13092

Berlin, Germany.

${ }^{\ddagger}$ G. S. Bhumbra and N. J. Moore have contributed equally to this work.
The fast inhibitory neurotransmitters glycine and GABA are co-localized in synaptic terminals of inhibitory interneurons in the spinal cord and co-released onto lumbar motoneurons in neonatal rats. We performed whole-cell voltage-clamp experiments on spinal cord preparations obtained from juvenile (P8-14) mice to determine whether inhibitory currents exhibited GABAergic components in motoneurons of animals of weight-bearing age. Subsequently we established whether or not GABA is co-released at glycinergic synapses onto motoneurons by determining if it conferred modulatory effects on the kinetics of glycinergic currents. Exponential fitting analysis showed that evoked and miniature inhibitory postsynaptic currents (IPSCs) were best-fitted with a single decay time constant. Responses recorded from connected interneuron-motoneuron pairs showed no effect of a benzodiazepine or a $\mathrm{GABA}_{A}$ receptor antagonist. Similarly IPSCs evoked by extracellular stimulation and miniature IPSCs were not affected by either agent, indicating the absence of codetection. Experimental manipulation of the relative content of pre-synaptic GABA and glycine conferred no effect on post-synaptic responses. It is thus unlikely that GABA is coreleased in biologically relevant amounts at glycinergic synapses onto lumbar motoneurons in mice of this age.

Keywords: glycine, GABA, spinal cord, motoneuron, co-release, mouse, patch clamp, paired recordings

\section{INTRODUCTION}

Fast inhibition in the nervous system is mediated by $\gamma$ aminobutyric acid (GABA) and glycine. Histological studies have shown that both neurotransmitters are present at synaptic terminals in the spinal cord (Bohlhalter et al., 1994; Taal and Holstege, 1994; Todd et al., 1996; Örnung et al., 1996). Recordings of miniature inhibitory post-synaptic currents (mIPSCs) from motoneurons (Jonas et al., 1998) have demonstrated that a high proportion of currents are mediated by both glycine and GABA. Since each mIPSC constitutes the post-synaptic response to an individual quantum of transmitter, the observation suggests that vesicles are loaded with both neurotransmitters which are released together; the notion of co-release was supported by similar observations in recordings from connected interneuron-motoneuron pairs.

Evidence of co-release has been reported in the brainstem of juvenile rats (O'Brien and Berger, 1999) and in the dorsal horn of adult rats (Chéry and De Koninck, 1999). GABA even modulates the glycinergic current and shortens the time constant of the decay phase (Lu et al., 2008) through its action as a partial agonist. Vesicular filling of both neurotransmitters is possible since the vesicular inhibitory amino acid transporter (VIAAT) is shared between GABA and glycine albeit with higher affinity for GABA (Wojcik et al., 2006).

Electrophysiological evidence of co-release on motoneurons (Jonas et al., 1998) however is based on experiments using preparations obtained mainly from young (P6-7) rats. Recordings from neurons in the dorsal horn (Keller et al., 2001) and from interneurons in the ventral horn (González-Forero and Alvarez, 2005) have identified populations of cells in which the extent of co-release decreases with age. In the adult rat, the majority of inhibitory inputs from IA interneurons and Renshaw cells onto motoneurons are glycinergic $(>80 \%)$ but a third of glycinergic terminals are also immunoreactive for the glutamic acid decarboxylase (GAD) enzyme (Alvarez et al., 2005). Since GAD is a marker for GABA synthesis, co-release may be a physiologically important mechanism of inhibition by a population of interneurons in the mature spinal cord.

In the present study, we recorded from motoneurons in spinal cord preparations obtained from mice of an age range (P8-14) in which they are almost fully weight-bearing. This is the latest developmental stage at which such recordings can be reliably obtained. The principal aim of the study was to ascertain whether GABA is released from pre-motor glycinergic interneurons and if it has any detectable effect on glycinergic inhibition of motoneurons. We thus performed experiments to detect the contribution of any GABAergic component in glycinergic inhibitory post-synaptic currents (IPSCs). Subsequently we manipulated the relative content of pre-synaptic GABA and glycine to determine if this had any modulatory effect on the kinetics of post-synaptic responses.

\section{MATERIALS AND METHODS}

Spinal preparations were extracted from P8-14 mice in which the enhanced green fluorescent protein (EGFP) is expressed under the control of the promoter of the neuronal glycine transporter Gly 2 
(Zeilhofer et al., 2005). The transgenic strain was used to assist identification of glycinergic neurons during paired recordings. All experiments were undertaken in accordance with the Animal (Scientific Procedures) Act (UK) 1986.

\section{SPINAL CORD PREPARATION}

Animals were anesthetized with urethane $1.8 \mathrm{mg} / \mathrm{kg}$ i.p. Intracardiac perfusion was performed with the same extracellular solution as used for the normal artificial cerebrospinal fluid (aCSF) perfused during records of composition (in $\mathrm{mM}$ ) $113 \mathrm{NaCl}, 3 \mathrm{KCl}$, $25 \mathrm{NaHCO}_{3}, 1 \mathrm{NaH}_{2} \mathrm{PO}_{4}, 2 \mathrm{CaCl}_{2}, 2 \mathrm{MgCl}_{2}$ and 11 D-glucose. Following decapitation, spinal cords were extracted using standard techniques (Beato, 2008). The procedure comprised of a ventral laminectomy followed by rapid dissection of the cord in oxygenated ice cold aCSF.

Tissue glue (Vetbond, WPI Scientific Instruments) was used to adhere the dorsal side of the cord to an agar block. Transverse slices of thickness $400 \mu \mathrm{m}$ were cut using a VT1000 vibrating microtome (Leica Microsystems) from the lumbar segments (L2-L5) in oxygenated ice cold solution containing (in mM) $130 \mathrm{~K}$-gluconate, 15 $\mathrm{KCl}$, 0.05 EGTA, 20 HEPES, 25 D-glucose, 3 kynurenic acid, and pH 7.4 (Dugué et al., 2005). For coronal sections, the agar block was glued horizontally to the base of the vibrating microtome and the dorsal horns were sliced away just dorsal to the central canal. Tissue preparations were incubated at $37^{\circ} \mathrm{C}$ in normal extracellular solution for approximately $45 \mathrm{~min}$ prior to experimentation. Experiments were performed at room temperature during which slices were continuously perfused at $5-8 \mathrm{ml} / \mathrm{min}$ with the aCSF bubbled with a $95 / 5 \% \mathrm{O}_{2} / \mathrm{CO}_{2}$ mixture.

\section{PATCH RECORDINGS}

Whole-cell voltage-clamp recordings from motoneurons were performed using an Axopatch 200B amplifier (Molecular Devices) and filtered with an eight-pole Bessel filter at $5 \mathrm{kHz}$. Both voltage and current signals were sampled at $50 \mathrm{kHz}$ using an Axon 1440A interface device (Molecular Devices) and the data were acquired using Clampex 10 software (Molecular Devices). Electrodes were pulled using a P-1000 Flaming/Brown micropipette puller (Sutter Instruments) from thick-walled borosilicate glass GC150F capillaries (Harvard Apparatus) to a resistance of $\sim 0.5 \mathrm{M} \Omega$. The tips were fire polished to final resistance of $\sim 1-1.5 \mathrm{M} \Omega$.

Electrodes were filled with an internal solution of composition (in $\mathrm{mM}$ ) $140 \mathrm{CsCl}, 4 \mathrm{NaCl}, 0.5 \mathrm{CaCl}_{2}, 10 \mathrm{HEPES}, 5$ EGTA, $2 \mathrm{Mg}$-ATP, QX-315 Br 3, pH 7.3 with $\mathrm{CsOH}$, and osmolarity of 290-310 mOsM. High intracellular chloride prolongs the decay phase of both glycinergic (Pitt et al., 2008) and GABAergic (Houston et al., 2009) currents through an interaction with amino acids in the pore-lining region of the channels (Moroni et al., 2011). A high chloride internal solution was chosen to improve the signalto-noise ratio and to allow direct comparison with previously published observations (Jonas et al., 1998).

Cells were visualized using an infrared differential interference contrast (DIC) optics on an Eclipse E600FN (Nikon) with a $40 \times$ water-immersion objective. Recordings were performed from motoneurons, identified by their position in the lateral motor column and soma diameter of at least $20 \mathrm{~mm}$ (Takahashi, 1992; Thurbon et al., 1998). Motoneurons were voltage-clamped at $-60 \mathrm{mV}$.
The series resistance of $4-10 \mathrm{M} \Omega$ was compensated by $60-80 \%$ and recordings were abandoned if it increased by more than $20 \%$. The typical motoneuron whole-cell capacitance of $\sim 200 \mathrm{pF}$ gave a corner frequency of $0.2-0.8 \mathrm{kHz}$.

During all recordings, the aCSF composition included $3 \mathrm{mM}$ kynurenic acid (Sigma) to block excitatory glutamatergic activity. Drugs were bath applied through the perfusion system as detailed below and in the Results section. Applied drugs included $5 \mu \mathrm{M}$ SR-95531 (Sigma), 0.3-2 $\mu \mathrm{M}$ strychnine (Sigma), $1 \mu \mathrm{M}$ diazepam (Sigma), $100 \mathrm{nM}$ tetrahydrodeoxycorticosterone (THDOC, Sigma), $20 \mathrm{mM}$ isoniazid (Sigma), $4 \mathrm{mM} \alpha-$ (methylamino) isobutyric acid (MeAIB, Sigma), $1 \mu \mathrm{M}$ (3S)-3-[[3[[4-(Trifluoromethyl)benzoyl]amino]phenyl]methoxy]-L-aspartic acid (TFB-TBOA, Tocris), and $2 \mathrm{mM}$ nipecotic acid (Tocris). Whenever we superfused isoniazid, $1 \mu \mathrm{M}$ CGP-555845- $\mathrm{HCl}$ was applied for the duration of the entire experiment to preclude activation of metabotropic GABA receptors.

In experiments where miniature inhibitory post-synaptic currents (mIPSCs) were recorded, $0.5 \mu \mathrm{M}$ tetrodotoxin (Tocris) was perfused after a whole-cell patch was established. This concentration of tetrodotoxin was maintained during control recordings but reduced to $0.2 \mu \mathrm{M}$ during drug application. At least 50 sweeps, of duration $9 \mathrm{~s}$, were recorded for each condition. After the control period, the agents $5 \mu \mathrm{M}$ SR-95531 or $0.3 \mu \mathrm{M}$ strychnine were included in the perfusate to isolate glycinergic or GABAergic mIPSCs respectively.

For experiments employing extracellular stimulation, we applied an electrical current through a patch pipette filled with normal aCSF using a constant current DS3 isolated stimulator (Digitimer). After patching a motoneuron in transverse slices, the stimulation electrode was maneuvered within the ventral region of Rexed lamina VIII, in the Renshaw cell area until a response could be elicited. Having established the minimum stimulation intensity required to evoke inhibitory post-synaptic currents (IPSCs) reliably, it was fixed at $\sim 1.5 \times$ threshold. For experiments performed on the coronal preparation, the stimulation electrode was placed in the ipsilateral lateral white matter at least two or three segments rostral or caudal to the motoneuron which was always recorded from the dorsolateral motor nucleus of L5.

\section{PAIRED RECORDINGS}

For paired recordings, we used an optical configuration that allowed simultaneous visualization of motoneurons and EGFP positive interneurons in transverse slices. Infrared-DIC transmitted light was collected through the back port of a beam splitter, while a laser scanning confocal D-Eclipse C1 camera (Nikon) mounted on the front port was used to collect light emitted from EGFP positive cells. Having established a stable whole-cell patch on a motoneuron, a second electrode of $\sim 6 \mathrm{M} \Omega$ filled with normal aCSF was introduced into the Renshaw area. Putative pre-synaptic neurons were patched in a loose cell-attached voltage-clamp configuration to stimulate the membrane and record evoked spikes (Barbour and Isope, 2000) using a $1-1.5 \mathrm{~V}$ voltage step of $20 \mu \mathrm{s}$ applied from an ELC-03X (NPI) amplifier.

Figure 1 illustrates how a connection was identified from post-synaptic responses of the motoneuron as IPSCs time-locked to evoked spikes. Typically, 1/100 of tested interneurons were 


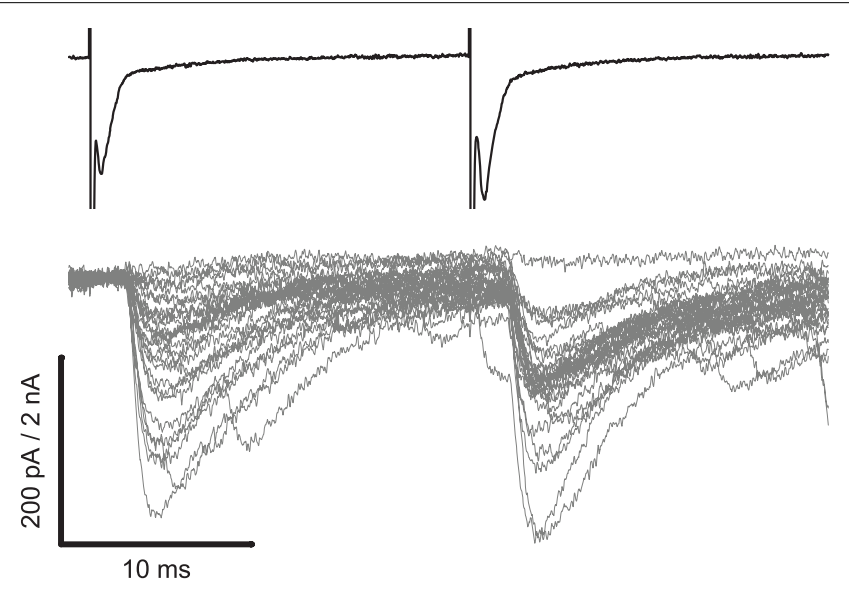

FIGURE 1 | Paired recordings with extracellular loose cell-attached stimulation of an interneuron can identify a connection with a motoneuron. The upper trace illustrates two voltage steps (see Materials and Methods) evoking individual spikes, clearly visible as downward deflections, from an interneuron patched in a loose cell-attached configuration. Post-synaptic responses of the motoneuron, illustrated in the lower trace, demonstrate a connection as IPSCs time-locked to the spikes. Following a $30 \mathrm{~ms}$ interval a second spike was evoked to potentiate the synapse and reduce the failure rate. connected to the recorded motoneuron. Since a number of synaptic connections exhibited a high failure rate, double stimulations were applied to induce paired-pulse facilitation to potentiate responses. Upon finding a connection, the pre-synaptic interneuron was re-patched using a $\sim 4 \mathrm{M} \Omega$ electrode containing an internal solution of composition (in $\mathrm{mM}$ ) K-gluconate 125, $\mathrm{KCl}$ 6, $\mathrm{CaCl}_{2}$ 2, HEPES 10, EGTA 10, Mg-ATP 2, pH 7.3 with $\mathrm{KOH}$, and osmolarity of 290-310 mOsM. In whole-cell current clamp, pre-synaptic cells were stimulated periodically every $9 \mathrm{~s}$ using an ELC-03X amplifier (NPI) by application of the minimum positive current required to evoke an action potential reliably.

\section{CONCENTRATION JUMPS}

Effects of the GABA-depleting agents on glycine receptors were investigated by performing concentration jump experiments on recombinant rat $\alpha 1 \beta$ glycine receptors, the adult isoform. Receptors were expressed in HEK293 cells using standard culture and transfection procedures (Burzomato et al., 2003). Concentration jumps were performed in an extracellular solution of composition (in $\mathrm{mM}$ ) $102.7 \mathrm{NaCl}, 20 \mathrm{Na}$ gluconate, $2 \mathrm{KCl}, 2 \mathrm{CaCl}_{2}, 1.2 \mathrm{MgCl}_{2}$, 10 HEPES, 14 D-glucose, 15 sucrose, and 20 TEACl, pH adjusted to 7.4 with $\mathrm{NaOH}$ (osmolarity $\sim 320 \mathrm{mOsM}$ ). Pipettes were filled with a high chloride solution containing (in $\mathrm{mM}$ ): $107.1 \mathrm{KCl}, 1$ $\mathrm{CaCl}_{2}, 1 \mathrm{MgCl}_{2}, 10 \mathrm{HEPES}, 11 \mathrm{EGTA}, 20 \mathrm{TEACl}$, and $2 \mathrm{Mg}$-ATP.

Outside-out patches were pulled and maneuvered toward a theta tube, of tip diameter of $150 \mu \mathrm{m}$, mounted on a piezo-stepper (Burleigh Instruments). While both barrels contained normal extracellular solution, one also included $1 \mathrm{mM}$ glycine. At least 5 fast applications of $1 \mathrm{~ms}$ were performed interleaved with $10 \mathrm{~s}$ periods for recovery. Before testing each GABA-depleting agent (i.e. isoniazid, MeAIB, TFB-TBOA, and nipecotic acid), the patch was first allowed to equilibrate with the new solution. Effects of fast application in control and test conditions on responses were measured using the changes in the peak current.

\section{ANALYSIS OF INHIBITORY CURRENTS}

Successfully evoked IPSCs were discriminated offline using Clampfit 10.2 (Molecular Devices) whereas mIPSCs were detected using WinEDR 3.2.4 (Strathclyde Electrophysiology Software). Both evoked IPSCs and mIPSCs were subjected to exponential fitting analysis using MATLAB 7 software (MathWorks). Events were excluded from the analysis if their amplitude was less than 3 standard deviations of the baseline noise, if there were overlapping events within $50 \mathrm{~ms}$, or if the asymptotic decay did not reach $10 \%$ of baseline. The Levenberg-Marquardt least-squares iterative algorithm was used to fit one or two exponential components to the decay phase of each post-synaptic current from 95 to $5 \%$ of peak amplitude:

$$
\begin{aligned}
& \hat{I}_{1}(t)=I_{0}+A e^{-\frac{t}{\tau}} \\
& \hat{I}_{2}(t)=I_{0}+A_{1} e^{-\frac{t}{\tau_{1}}}+A_{2} e^{-\frac{t}{\tau_{2}}}
\end{aligned}
$$

The fit was selected on the basis of the F-test statistics for the two curves with $F>4.8$ as a threshold for favoring the second. Since the decay constants were normally distributed, they were amenable to parametric test statistics. Comparisons across treatments were undertaken using one-way ANOVA $F$ statistics, with post-hoc tests based on Student's $t$ statistics employing Bonferroni's correction for multiple comparisons. Summary data are presented as mean \pm SEM. To confirm successful detection of mixed inhibitory currents, we performed exponential fitting analysis on mIPSCs recorded from motoneurons $(n=5)$ of neonatal (P0-3) mice. Under control conditions, bi-exponential fits consistently represented a proportion of events $(14.5 \pm 4.5 \%)$ that was substantially reduced (to $4.7 \pm 1.0 \%$ ) in the presence of $5 \mu \mathrm{M}$ SR-95531.

Since mIPSCs were always recorded in the presence of kynurenic acid, inward currents could only have resulted from GABAergic or glycinergic events. We thus quantified the overall inhibitory drive for each sweep by evaluation of the integral of 
the entire current trace per unit of time. Using pharmacological isolation of GABAergic or glycinergic events, we used the integral to estimate their relative contributions to overall inhibitory drive.

Prior to integral estimation however we first corrected for slow baseline drifts. We used a low-pass filter at $0.5 \mathrm{~Hz}$ that canceled completely the fast-rising events associated with synaptic activity and subtracted the result from the original signal to obtain a drift-free trace. Since the subtraction could impose a non-zero center for the baseline, it was necessary to eliminate any offsetting effects on the integral evaluation by a further subtraction of this bias. We estimated the bias using the mode of the data, which was evaluated by convolution with a Gaussian kernel of a standard deviation $\sigma n^{-0.3}$, where $n$ is the size and $\sigma^{2}$ is the variance of the data (Bhumbra and Dyball, 2010).

\section{RESULTS}

\section{EVOKED IPSCs EXHIBITED NO CO-DETECTION}

Simultaneous recordings of interneurons and motoneurons were obtained from eight connected pairs. In all cases, strychnine completely abolished evoked IPCSs (data not shown). In a subset of four, responses were tested with bath application of diazepam and gabazine for exponential fitting analysis of the decay phase. Evoked IPSCs were best-fitted with a single exponential in all cases under control conditions $(n=203)$ and in the presence of diazepam $(n=143)$. Across all four pairs, the mean of the averaged time constant $\tau$ fitted for all sweeps was $3.3 \pm 0.2 \mathrm{~ms}$. The lack of a second component to the exponential fits and the very short mean time constant are consistent with a purely glycinergic component to evoked currents.

Pharmacological modulation of any GABAergic component with diazepam and gabazine confirmed the lack of co-detection of GABA with glycine. A representative example of a paired recording for the different drug treatments is illustrated in Figure 2A. Responses of the motoneuron showed no effect by application of $1 \mu \mathrm{M}$ diazepam or $5 \mu \mathrm{M}$ SR-95531 of evoked IPSCs. By contrast, responses were abolished after application of $2 \mu \mathrm{M}$ strychnine.

Group data for the four connected pairs are illustrated in Figure 2B. For three of the pairs, one-way ANOVA statistics showed no statistically significant effect of the treatments on the decay constant $(F \leq 0.11, P \geq 0.523)$. In the fourth pair, the test statistic was significant $(F=19.3, P<0.001)$ as result of a small decrease in the time constant in the presence of diazepam $(\tau=3.2 \pm 0.1 \mathrm{~ms})$ compared to control ( $\tau=3.9 \pm 0.1 \mathrm{~ms}, t=4.64, P<0.001)$. A decrease in the time constant however is not consistent with the effect of diazepam enhancing and prolonging the GABA component of evoked IPSCs.

Comparisons of mean amplitudes to those observed in control conditions showed a small decrease in the presence of diazepam $(82 \pm 11 \%)$ and SR-95531 (93 $\pm 8 \%)$. Since superfusion of either agent resulted in modest decreases, we attributed the progressive
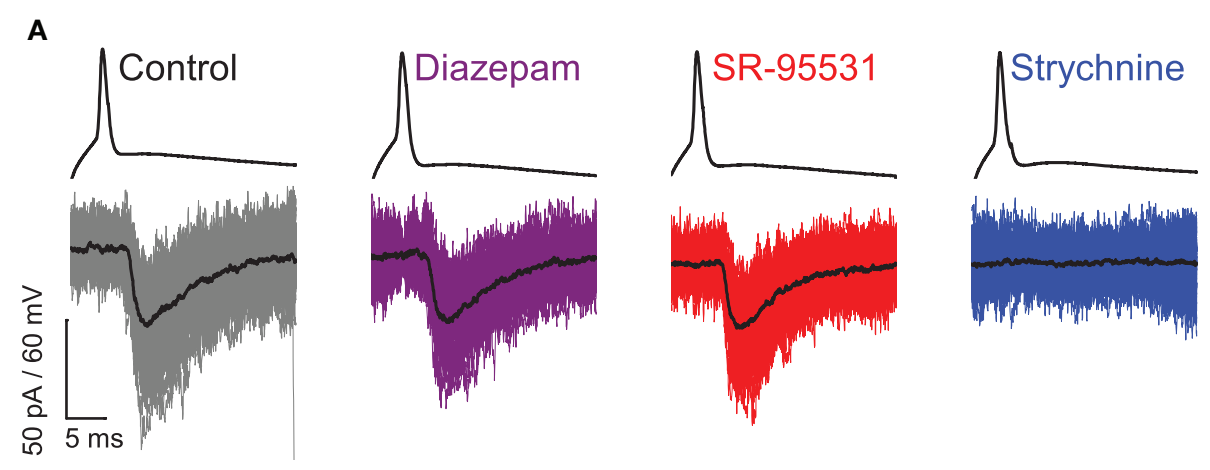

B

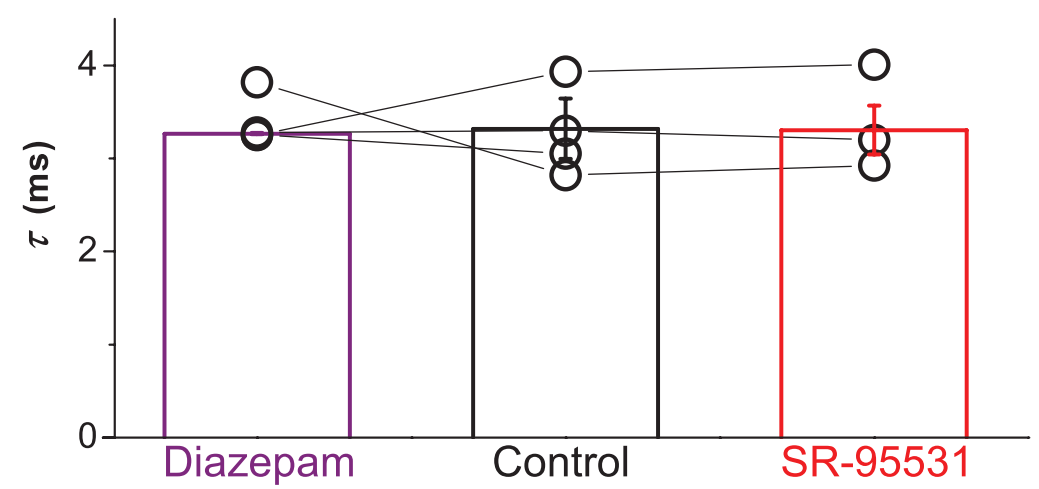

FIGURE 2 | Paired recordings showed no contribution of GABA to evoked IPSCs. Traces in (A) illustrate responses from a connected pair representing a single spike elicited in the interneuron above and the evoked IPSCs recorded from the motoneuron below with the mean current overlayed in black. Bath application of neither $1 \mu \mathrm{M}$ diazepam (purple) nor $5 \mu \mathrm{M}$ SR-95531 (red) modulated the time constant of evoked IPSCs whereas $2 \mu \mathrm{M}$ strychnine (blue) abolished all responses. Group data from four connected pairs are represented in graph (B), which illustrates no significant effect of diazepam (purple) or SR-95531 (red) on the time constant (see text). Error bars indicate mean \pm SEM. 
attenuation of IPSCs to the inevitable run down of neurotransmitter due to dialysis inherent to paired recordings (Diana and Marty, 2003). Analysis of the paired recordings thus indicated that there is no detectable GABAergic component the evoked IPSCs.

Paired recordings were performed on transverse slices and thus were selective for horizontal rather than vertical connections. It is possible that axon terminals from horizontal and vertical projections differ in their neurotransmitter content (Liu et al., 2010) or are apposed to post-synaptic membranes with different compositions of receptors. We thus investigated the effects of extracellular stimulation of ascending or descending connections on evoked IPSCs using the coronal preparation.

Seven motoneuronal recordings were obtained from coronal preparations while stimulating ascending or descending projections. Evoked IPSCs were best-fitted with a single exponential in all cases under control conditions $(n=223)$ and in the presence of diazepam $(n=223)$. Across all cells, the mean of the averaged time constant $\tau$ fitted for all sweeps was $5.8 \pm 0.9 \mathrm{~ms}$. The lack of a second component to the exponential fits and the very short mean time constant are consistent with a purely glycinergic component to evoked currents.

Superfusion of diazepam or gabazine confirmed the lack of codetection of GABA with glycine. A representative example of a evoked IPSCs recorded during different drug treatments is illustrated in Figure 3A. Responses of the motoneuron showed no effect by application of $1 \mu \mathrm{M}$ diazepam or $5 \mu \mathrm{M}$ of evoked IPSCs. By contrast, responses were abolished after application of $1 \mu \mathrm{M}$ strychnine.

Group data for the seven motoneurons are illustrated in Figure 3B. Paired $t$-test statistics evaluated from the average fitted time constant for each cell across all seven motoneurons confirmed no significant effect on the decay kinetics of evoked IPSCs by diazepam $(\tau=6.1 \pm 0.9 \mathrm{~ms}, t=-1.51, P=0.183)$ or SR-95531 $(\tau=5.7 \pm 1.0 \mathrm{~ms}, t=-1.04, P=0.346)$. Analysis of the recordings obtained from coronal preparations thus indicated that there is no detectable GABAergic component in IPSCs evoked by simulation of ascending or descending projections.

To investigate putative GABAergic components of currents mediated by diazepam-insensitive $\mathrm{GABA}_{\mathrm{A}}$ receptors, we recorded evoked responses from four motoneurons in transverse slices during bath application of the $200 \mathrm{nM}$ THDOC. Group results are illustrated in Figure 3C. In comparison to control conditions ( $\tau=5.5 \pm 0.3 \mathrm{~ms}$ ), paired $t$-test statistics showed no significant effect of THDOC on the time constant $(\tau=5.6 \pm 0.3 \mathrm{~ms}$, $t=-0.47, P=0.672)$.

\section{RECORDINGS OF mIPSCS SHOWED NO CO-DETECTION}

Since neither paired recordings nor extracellular stimulation showed demonstrable GABA co-detection in evoked responses, we investigated whether there were any GABAergic components
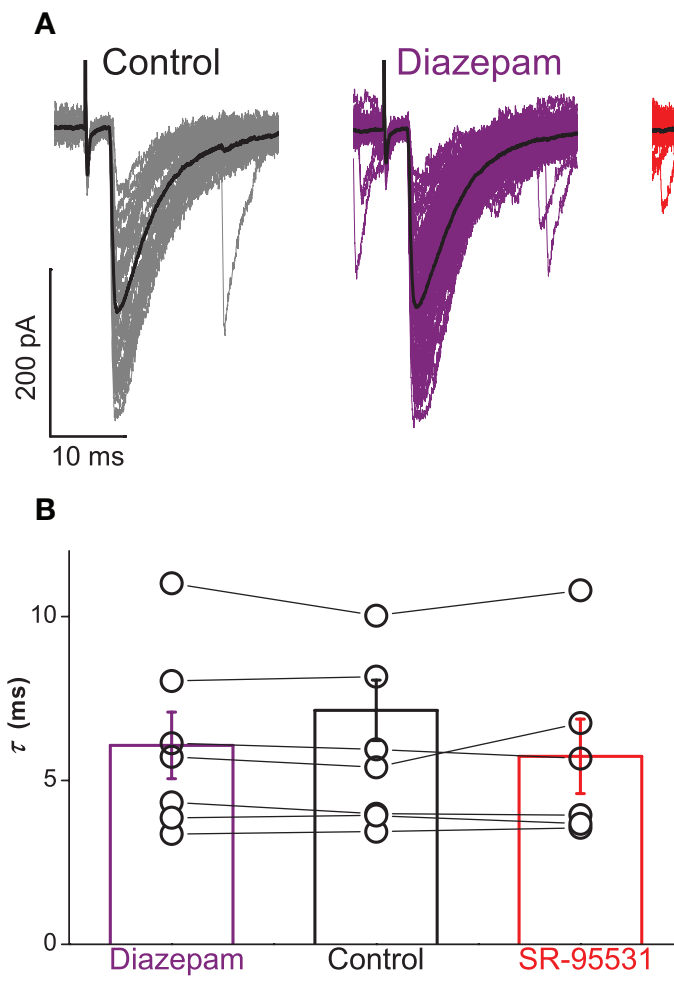

FIGURE 3 | Extracellular stimulation of ascending and descending projections showed no contribution of GABA to evoked IPSCs. Traces in (A) illustrate responses of a motoneuron with the mean current overlayed in black. Bath application of neither $1 \mu \mathrm{M}$ diazepam (purple) nor $5 \mu \mathrm{M}$ SR-95531 (red) modulated the time constant of evoked IPSCs whereas $2 \mu \mathrm{M}$ strychnine
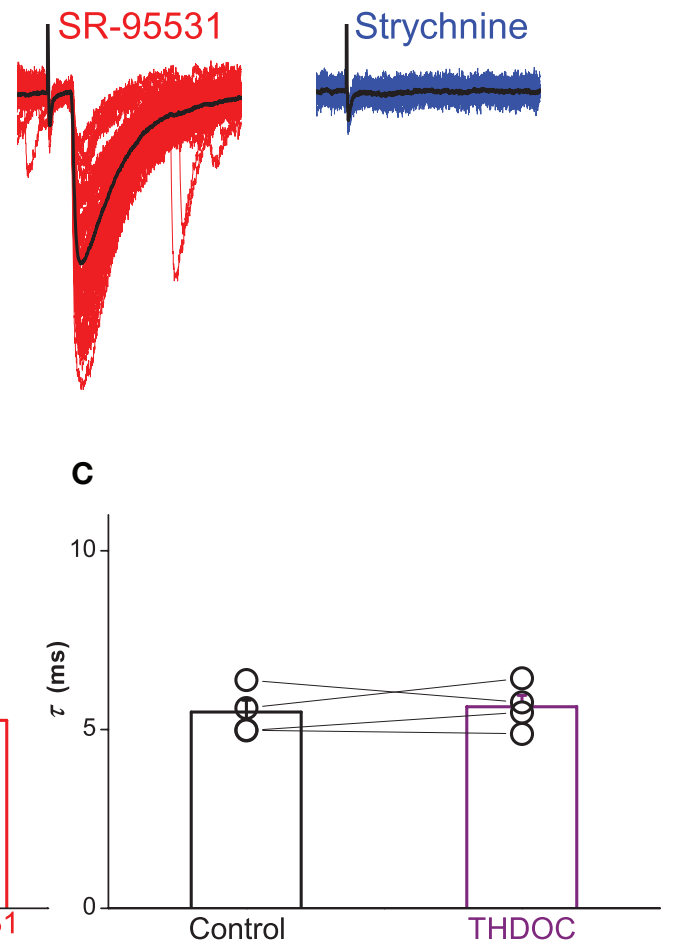

(blue) abolished all responses. Group data for all motoneurons are represented in graph (B), which illustrates no significant effect of diazepam (purple) or SR-95531 (red) on the time constant. Graph (C) shows no significant effect of $200 \mathrm{nM}$ THDOC (purple) on the time constant of responses evoked in transverse slices (see text). 
in miniature IPSCs. Miniature currents were recorded from seven motoneurons of which six were tested with $5 \mu \mathrm{M}$ SR-95531 and four were tested with $1 \mu \mathrm{M}$ diazepam. Exponential fitting analysis showed that the decay phase of currents were best-fitted with a single exponential in control conditions $(n=1398)$ and in the presence of diazepam $(n=992)$. Across all motoneurons, the mean of the averaged time constant $\tau$ fitted for all mIPSCs under control conditions was $6.0 \pm 0.9 \mathrm{~ms}$. The lack of a second component to the exponential fits and the short mean time constant are consistent with purely glycinergic miniature currents.

Pharmacological modulation of any GABAergic component with diazepam and gabazine confirmed the lack of co-detection of GABA with glycine. A representative example of mIPSCs recorded from a motoneuron during different drug treatments is illustrated in Figure 4A. Exponential fitting analysis showed no effect of diazepam or SR-95531 on the evoked IPSCs.

Group data for the mIPSCs recorded from all the cells are illustrated in Figure 4B. The mean relative amplitude of the currents compared to control conditions was not changed in the presence of diazepam $(99.4 \pm 5.0 \%)$ or SR-95531 (99.1 $\pm 6.9 \%)$. There was also no significant change on the mean fitted time constant with diazepam $(\tau=6.2 \pm 1.3 \mathrm{~ms}$, paired $t=-0.57, P=0.607)$ or SR-95531 ( $\tau=5.9 \pm 0.8 \mathrm{~ms}$, paired $t=0.50, P=0.618)$.

Since the experiments were performed at room temperature, the apparent lack of a second component in mIPSCs could have resulted from a reduction in GABA synthesis from glutamate due to a decrease in the activity of glutamate transporters. We thus recorded mIPSCs from five motoneurons at $32^{\circ} \mathrm{C}$ under control conditions and in the presence of diazepam. Exponential fitting analysis showed that the decay phase of currents were best-fitted with a single exponential before $(n=4111)$ and after $(n=2972)$ diazepam administration. As expected for recording at higher temperatures, the mean decay constant was markedly short in control conditions $(\tau=2.7 \pm 0.2 \mathrm{~ms})$. Superfusion of diazepam nevertheless had no significant effect on the fitted time constant ( $\tau=3.1 \pm 0.5 \mathrm{~ms}$, paired $t=-1.06, P=0.348$ ).

The short time constant and the minimal effects of diazepam and SR-95531 suggested that glycinergic contribution to inhibitory inputs were substantially greater than the GABAergic component notwithstanding the absence of co-detection. Their relative contributions in mIPSC activity at room temperature were evaluated using integral analysis for the recordings in which each component was pharmacologically isolated. A representative example of such a recording during different drug administrations is illustrated in Figure 4C. While application of $5 \mu \mathrm{M}$ SR-95531 had no demonstrable effect on the activity of mIPSCs, they were virtually abolished by $0.3 \mu \mathrm{M}$ strychnine. The example thus illustrates a substantially greater contribution of a glycinergic inputs compared to inhibition mediated by GABA.

Group data for the mIPSC integral analysis for the six cells recorded are illustrated in Figure 4D. In comparison to control conditions, the overall inhibitory drive did not change in presence of SR-95531 in five cells $(99.0 \pm 6.4 \%$ of control) whereas it was significantly reduced in only one (to $45.0 \pm 25.7 \%$ ). By

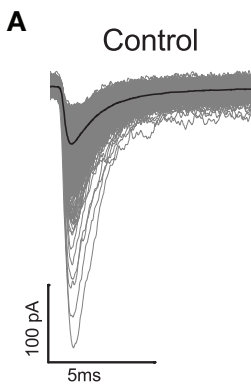

C
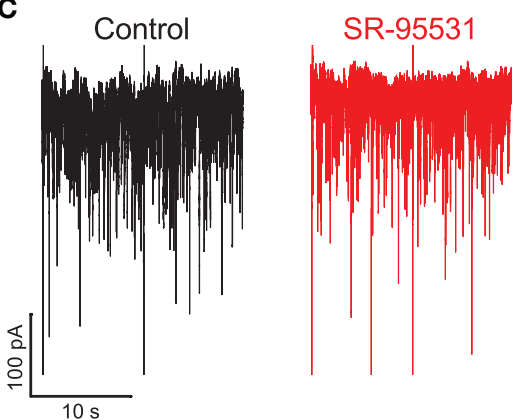

FIGURE 4 | Neither diazepam nor SR-95531 changed the activity of mIPSCs although they were substantially attenuated by strychnine.

Traces in (A) illustrate mIPSCs recorded from the motoneuron with the mean current overlayed in black, demonstrating no detectable effect of the two agents. Group data from all recorded motoneurons are represented in graph (B), which illustrates no significant effect of diazepam (purple) or SR-95531 (red) on the relative amplitude or time constant of mIPSCs. Traces in (C)
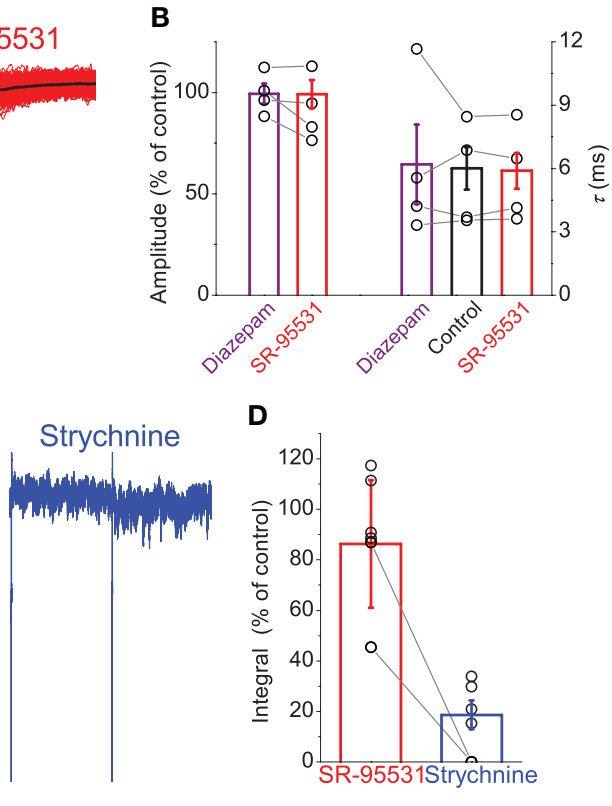

D

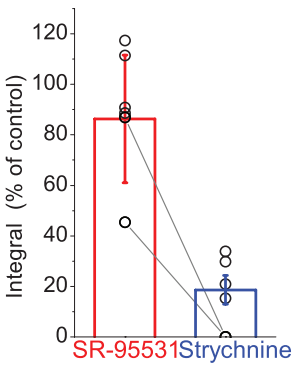

illustrate recordings from a neuron that were subjected to integral analysis (see text; the biphasic currents resulting from voltage steps are truncated and were excluded from the analysis) to quantify overall inhibitory drive, which was minimally affected by application of SR-95531 (red), but heavily reduced by strychnine (blue). Group data illustrated in graph (D) show that overall inhibitory drive is not significantly affected by SR-95531 (red) but substantially attenuated by strychnine (blue) and abolished in some cases. 
contrast, out all the seven cells tested with strychnine, activity was completely abolished in three cells and the remaining four exhibited a profound suppression of inhibitory activity following drug administration (to $24.9 \pm 4.1 \%$ ). The integral analysis thus demonstrates a substantial predominance of a glycinergic component to inhibitory inputs in comparison to GABAergic contributions.

\section{GABA DEPLETION DID NOT AFFECT EVOKED IPSCs}

While the single exponential profile of IPSCs and pharmacological isolation of a GABAergic component demonstrated no co-detection, it did not preclude possible co-release onto a postsynaptic membrane with a dearth of GABA receptors. Since GABA however is a partial agonist of post-synaptic glycine receptors, its co-release could attenuate glycinergic currents and would shorten their time constant (Lu et al., 2008). Glutamate uptake contributes to GABA synthesis (Mathews and Diamond, 2003) by the action of GAD. Glutamate is either transported into cells or synthesized from glutamine by glutaminase.

In order to deplete GABA, we used 4 mM MeAIB to block glutamine uptake (Varoqui et al., 2000), $1 \mu \mathrm{M}$ TFB-TBOA to block glutamate uptake (Shimamoto et al., 1998), and $20 \mathrm{mM}$ isoniazid to inhibit GAD (De Koninck and Mody, 1997) thus depleting upstream substrates of GABA and directly inhibiting its synthesis from glutamate. GABA uptake into cells was blocked using $2 \mathrm{mM}$ nipecotic acid. During recordings of glycinergic currents from voltage-clamped motoneurons, responses evoked from extracellular stimulation in the Renshaw cell area were attenuated by $~ 70 \%$ following administration of isoniazid, MeAIB, TFB-TBOA, and nipecotic acid (data not shown). Since the decrease in peak current was observed within $5 \mathrm{~min}$ of drug application, the reduction in response was unlikely to have resulted from perturbations in metabolic processes but as a consequence of direct action of the four drugs on the receptors mediating post-synaptic currents. We thus sought to identify which of the GABA-depleting agents confer the least direct action on glycinergic receptors using concentration jumps.

Figure 5 illustrates responses to fast glycine applications on outside-out patches pulled from HEK293 cells expressing recombinant $\alpha 1 \beta$. Under control conditions (Figure 5A), concentration jumps showed uniform responses. In the illustrated example, bath application of $20 \mathrm{mM}$ isoniazid resulted in an attenuation of the current.

Group data illustrated in Figure 5B illustrates that all GABAdepleting agents decreased the peak current, which were observed within $5 \mathrm{~min}$ of administration. Reductions in mean amplitude were substantial following application of $4 \mathrm{mM}$ MeAIB $(25.5 \pm 3.9 \%, n=7), 1 \mu \mathrm{M}$ TFB-TBOA $(34.4 \pm 9.8 \%, n=5)$, and $2 \mathrm{mM}$ nipecotic acid $(29.0 \pm 4.2 \%, n=7)$. The comparatively modest attenuation associated with isoniazid $(14.0 \pm 3.0 \%, n=5)$ thus identified this agent as our choice of drug for depleting GABA.

We confirmed the efficacy of GABA depletion by isoniazid using extracellular stimulation to evoke IPSCs recorded from whole-cell voltage-clamped motoneurons during administration of $20 \mathrm{mM}$ isoniazid in the presence of $1 \mu \mathrm{M}$ strychnine to isolate GABAergic currents. A representative example of the progressive attenuation in currents is illustrated in Figures 6A-C. A plot of the corresponding changes in amplitude (Figure 6D) shows that the attenuation was substantial and occurred over a period of 30-45 min, demonstrating an effect more compatible with influences on metabolic processes rather than a direct action on post-synaptic receptors.

Group data represented in Figure 6E illustrates the relative change in evoked IPSC amplitude when comparing the peak currents observed 30-45 min after isoniazid administration with those recorded during the control period. In all cases $(n=4)$ there was an attenuation in the response, with an average reduction
A

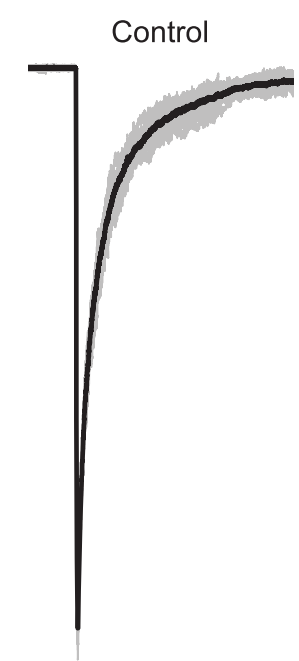

Isoniazid

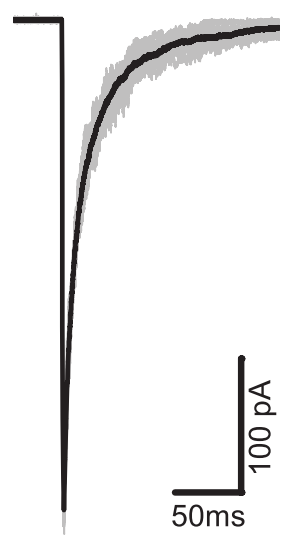

B

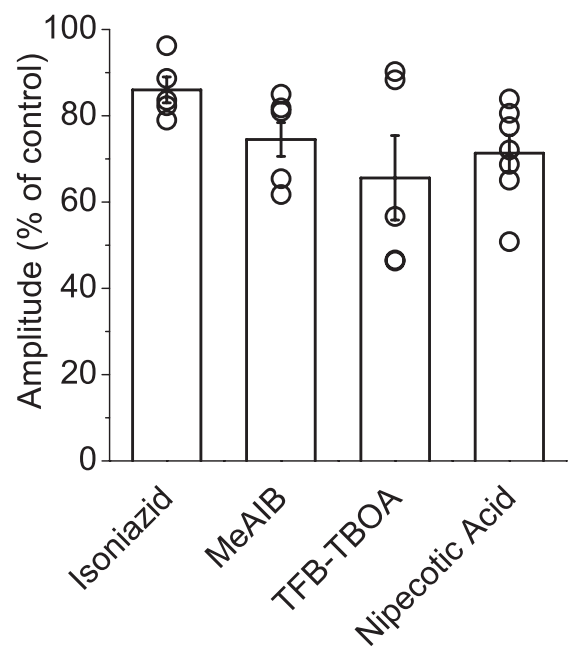

reduction in the current is observed in the presence of $20 \mathrm{mM}$ isoniazid. Graph (B) illustrates isoniazid attenuated the current to the least extent (see text) compared to $4 \mathrm{mM}$ MeAIB, $1 \mu \mathrm{M}$ TFB-TBOA, and $2 \mathrm{mM}$ nipecotic acid
FIGURE 5 | Fast glycine application experiments showed that isoniazid administration produced the least attenuation in current among the GABA-depleting agents. Traces in (A) illustrate an example in which a small 


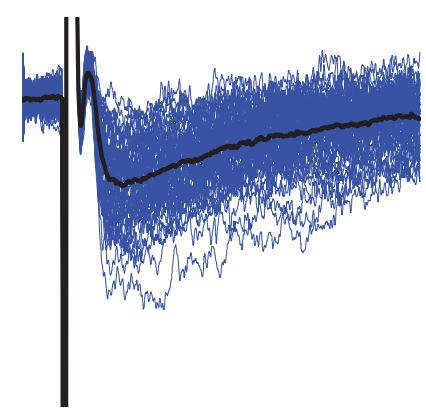

D

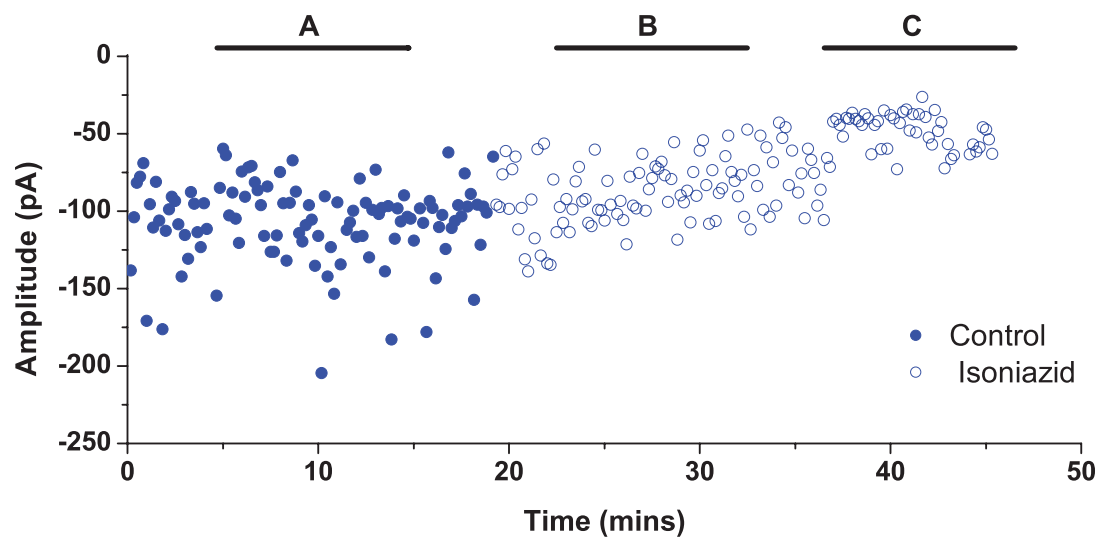

C
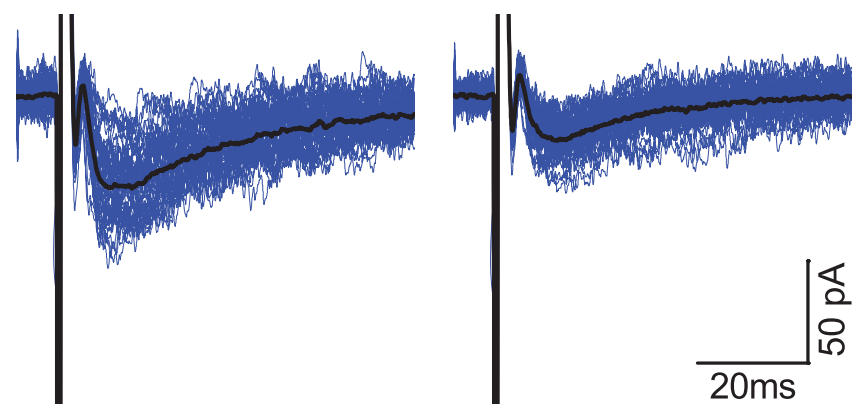

E

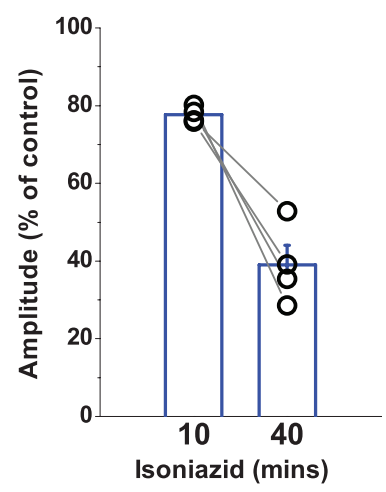

FIGURE 6 | Application of $20 \mathrm{mM}$ isoniazid depleted vesicular GABA over a period of $\mathbf{3 0 - 4 5} \mathbf{~ m i n}$. In the presence of $1 \mu \mathrm{M}$ strychnine, IPSCs were evoked by extracellular stimulation. The traces illustrate an example of a recording of post-synaptic responses during the control period [trace $(\mathbf{A})]$ and two epochs following isoniazid superfusion [traces (B) and (C)]. Mean responses are overlayed in black. Graph (D) plots the changes in amplitude, illustrating the time periods corresponding to (A-C), and shows a gradual but substantial reduction in current. The group data represented in graph (E) illustrates that evoked IPSCs in all motoneurons were progressively attenuated during the $\sim 40$ min of isoniazid superfusion. of $61 \pm 5 \%$. The results thus confirm that application of $20 \mathrm{mM}$ isoniazid depletes vesicular GABA over a period of 30-45 min.

Since co-released vesicular GABA would hasten the decay of glycinergic currents (Lu et al., 2008), then any GABA-mediated effect on evoked IPSCs would be perturbed by isoniazid administration over a time scale of $30-45$ min during which the decay would become progressively longer. In the absence of strychnine, evoked IPSC recordings were obtained during application of $20 \mathrm{mM}$ isoniazid. A representative example is illustrated in Figures $7 \mathrm{~A}-\mathrm{C}$, which shows only a modest reduction in the size of the current. The plot of corresponding changes in amplitude (Figure 7D) however shows the attenuation was modest and occurred over a period of $\sim 10 \mathrm{~min}$, demonstrating an effect more compatible with direct action on post-synaptic receptors rather than on metabolic processes.

Group data $(n=6)$ are illustrated as graphs showing the effect on the relative amplitude (Figure 7E) of isoniazid superfusion after $10 \mathrm{~min}$ and after an hour. While a modest attenuation of $13.5 \pm 6.6 \%$ in the current amplitude was observed after $10 \mathrm{~min}$, the reduction in amplitude after an hour was only $15.8 \pm 7.6 \%$. Since there was only a small fast attenuation, the changes in relative amplitude demonstrate an effect more compatible with direct action on post-synaptic receptors rather than on metabolic processes. Comparison of time constants of evoked responses in control conditions with those after an hour of isoniazid superfusion (Figure 7F) showed no significant change from $\tau=5.8 \pm 0.8$ to $\tau=5.8 \pm 0.8 \mathrm{~ms}$ (paired $t=-0.05, P=0.963$ ). The results indicate that little or no GABA is co-released in glycinergic synapses investigated in the present study.

\section{GLYCINE LOADING DID NOT AFFECT EVOKED IPSCS}

Since GABA depletion did not affect the kinetics of evoked IPSCs, we investigated the effects of loading glycine into pre-synaptic vesicles using paired recordings. Addition of $20 \mathrm{mM}$ glycine to the internal solution of the pipette used to patch the interneuron would favor glycine loading compared to GABA. Any changes to the neurotransmitter content of pre-synaptic vesicles would be detected as a progressive change in post-synaptic responses over time after establishing a whole-cell configuration for the interneuron. After stabilization of the current clamp on the pre-synaptic neuron, trains of 1000 spikes at $50 \mathrm{~Hz}$ were delivered to deplete vesicules every $10-15 \mathrm{~min}$.

There were however no progressive changes in evoked currents recorded from connected pairs $(n=4)$. In the representative example illustrated in Figure 8A, IPSCs evoked immediately upon establishing a whole-cell configuration were not manifestly 


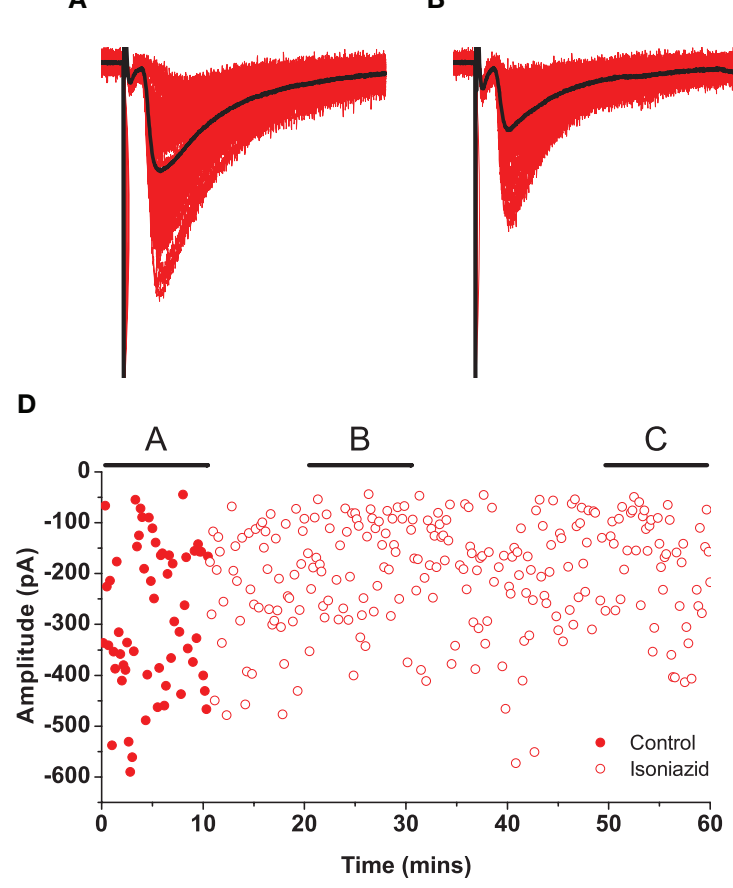

C

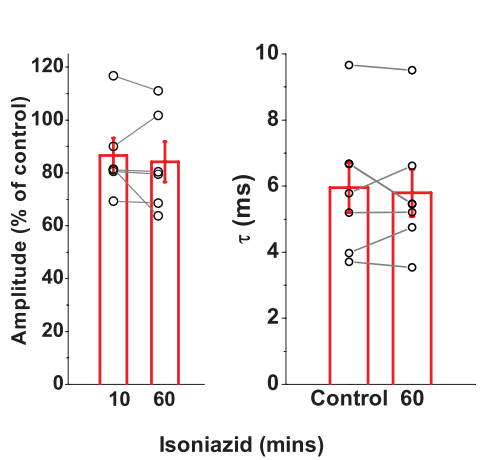

FIGURE 7 | Effects on glycinergic IPSCs by application of $20 \mathrm{mM}$ isoniazid were not compatible with vesicular GABA depletion. The traces illustrate an example of a recording of post-synaptic responses during the control period [trace (A)] and two epochs following isoniazid superfusion [traces (B) and (C)]. Mean responses are overlayed in black. Graph (D) plots the changes in amplitude, illustrating the time periods corresponding to (A-C), and shows a modest but rapid reduction in current. The group data for all motoneurons shows that the attenuation of evoked IPSCs [graph (E)] after $1 \mathrm{~h}$ was no greater than that observed after $\sim 10 \mathrm{~min}$, and that isoniazid superfusion had no systematic effect on the decay time constant [graph (F)]. different to those recorded after $\sim 45 \mathrm{~min}$. This suggests that the vesicle content was not affected by loading the pre-synaptic terminal with glycine.

Group data for the time constants (Figure 8B) and amplitudes (Figure $\mathbf{8 C}$ ) are illustrated as graphs over time. There was no appreciable effect on the decay constant $\tau$, with a mean in control of $4.8 \pm 0.4 \mathrm{~ms}$, and of $4.6 \pm 0.4 \mathrm{~ms}$ after $\sim 45 \mathrm{~min}$ of recording. A Spearman rank test statistic showed in three connected pairs no changes in the decay constant over time $(|r| \leq 0.13, P \geq 0.183)$, whereas the decrease observed in the fourth $(r=-0.39, P<0.001)$ was incompatible with a reduction in vesicular GABA content. Relative mean amplitudes decreased to $98.9 \pm 11.7 \%$ thus reflecting no changes on the magnitude of evoked responses beyond a possible counteraction of the effects of run-down associated with dialysis. The group data thus suggests that even if GABA was present in pre-synaptic vesicles, its influence was too small to detect.

\section{DISCUSSION}

Analysis of our initial paired recordings identified individual glycinergic synaptic connections in which GABA is not codetected. Co-detection was also absent in responses to extracellular stimulation of glycinergic projections in the coronal preparation. There was no demonstrable GABAergic contribution to the substantially larger number of inputs associated with the mIPSCs recorded from motoneurons in transverse slices. Our initial results therefore show that glycinergic synapses onto lumbar motoneurons have no ostensible $\mathrm{GABA}_{\mathrm{A}} \mathrm{R}$-mediated component.

Mixed evoked and miniature IPSCs with pharmacologically isolable GABAergic and glycinergic components have however been observed in recordings from lumbar motoneurons (Jonas et al., 1998). While our results may appear to contrast with those previously reported, the apparent discrepancy is most likely to have arisen as a result of differences in the age and species used for recordings. We used P8-14 mice whereas (Jonas et al., 1998) recorded mainly from $\mathrm{P} 6-7$ rats. Mice are already weight-bearing at P8 whereas rats are not until P12. The dissimilar findings may thus reflect the different stages of maturation of the spinal cord for the two animals.

While mIPSC recordings from interneurons in the ventral horn have exhibited co-detection in neonatal rats (González-Forero and Alvarez, 2005), the $\mathrm{GABA}_{\mathrm{A}} \mathrm{R}$-mediated component is diminished specifically in non-Renshaw cells in juveniles (from P9). In the dorsal horn of the rat (Keller et al., 2001), the extent of co-detection also decreases with age and is absent altogether in the mature state $(\geq \mathrm{P} 21)$. Since our exponential fitting analysis showed neither a second component in evoked or miniature IPSCs nor any pharmacologically isolable GABAergic contribution in evoked responses, co-detection must already be negligible in lumbar motoneurons of P8-14 mice.

Notwithstanding the absence of co-detection, the mIPSC analysis demonstrates that the glycinergic contribution to inhibitory inputs in juvenile mice is substantially greater than the GABAergic 


\section{A}
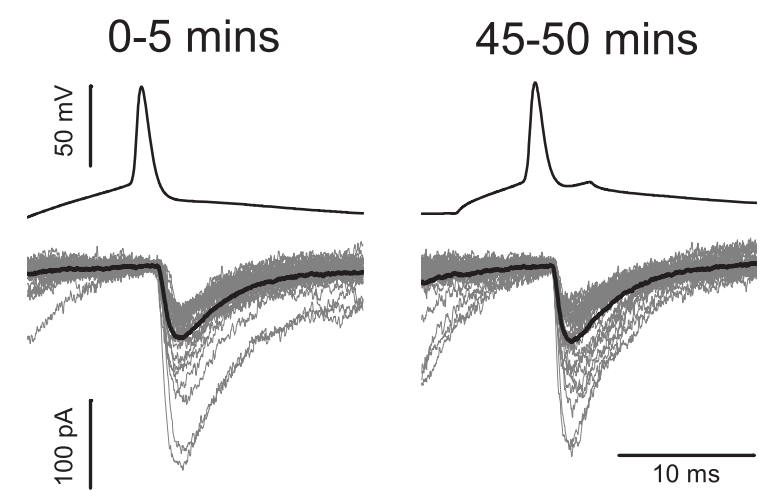

FIGURE 8 | Loading the pre-synaptic cell with $20 \mathrm{mM}$ glycine during paired recordings resulted in no progressive changes in post-synaptic responses. Traces in (A) illustrate an example of interneuronal spikes and motoneuronal responses for the first 5 and $45 \mathrm{~min}$ after rupturing the

\section{B}

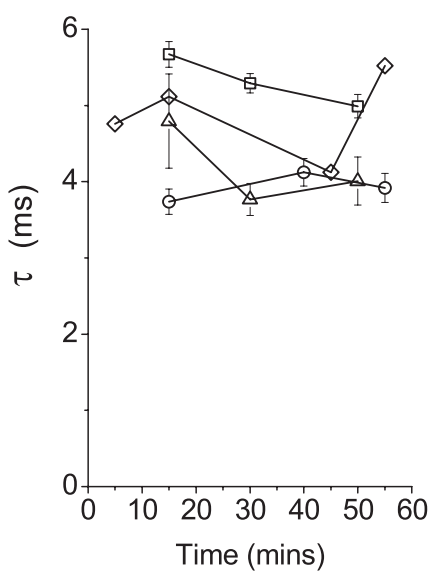

C

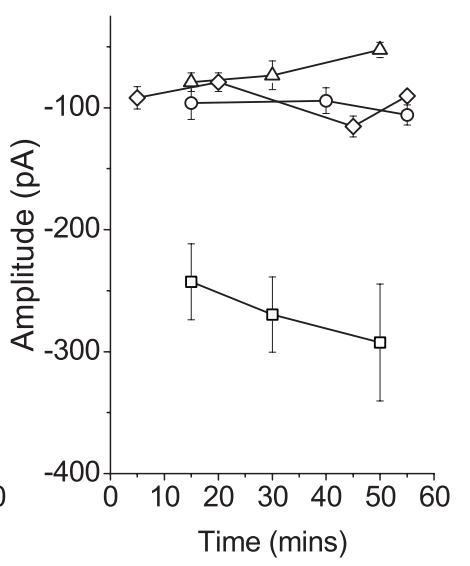

membrane of the pre-motor interneuron. Group data $(n=4)$ is illustrated graphically to show changes in the decay constant (B) and amplitude (C), neither of which showed systematic changes during the course of the loading glycine into the pre-synaptic vesicles. component. In the ventral horn there is a postnatal shift in the distribution of mIPSCs from GABAergic to glycinergic inhibition (Gao et al., 2001), a reduction in immunoreactivity of GABA and GAD (Ma et al., 1992), and a decrease in $\mathrm{GABA}_{\mathrm{A}} \mathrm{R}$ expression (Ma et al., 1993).

We investigated whether the lack of a GABAergic component in the evoked and miniature IPSCs resulted from a paucity of co-released GABA or post-synaptic $\mathrm{GABA}_{\mathrm{A}}$ receptors by experimental manipulation of the relative pre-synaptic content of GABA and glycine. Currents evoked by extracellular stimulation were not affected by pre-synaptic depletion of GABA. Paired recordings showed no modulatory effect on post-synaptic responses while

\section{REFERENCES}

Alvarez, F. J., Jonas, P. C., Sapir, T., Hartley, R., Berrocal, M. C., Geiman, E. J., Todd, A. J., and Goulding, M. (2005). Postnatal phenotype and localization of spinal cord $\mathrm{v} 1$ derived interneurons. J. Comp. Neurol. 493, 177-192.

Barbour, B., and Isope, P. (2000). Combining loose cell-attached stimulation and recording. J. Neurosci. Methods 103, 199-208.

Beato, M. (2008). The time course of transmitter at glycinergic synapses onto motoneurons. J. Neurosci. 28, 7412 .

Bhumbra, G. S., and Dyball, R. E. J. (2010). Reading between the spikes of the hypothalamic neural code. J. Neuroendocrinol. 22, 1239-1250.

Bohlhalter, S., Mohler, H., and Fritschy, J. M. (1994). Inhibitory neurotransmission in rat spinal cord: colocalization of glycine-and $\mathrm{GABA}_{\mathrm{A}}$ receptors at gabaergic synaptic contacts demonstrated by triple immunofluorescence staining. Brain Res. 642, 59-69.

Burzomato, V., Groot-Kormelink, P. J., Sivilotti, L. G., and Beato, M. (2003). Stoichiometry of recombinant heteromeric glycine receptors revealed by a pore-lining region point mutation. Recept. Channels 9, 353-361.

Chéry, N., and De Koninck, Y. (1999). Junctional versus extrajunctional glycine and gabaareceptor-mediated ipscs in identified lamina $i$ neurons of the adult rat spinal cord. $J$. Neurosci. 19, 7342-7355.

De Koninck, Y., and Mody, I. (1997). Endogenous GABA activates smallconductance $\mathrm{K}+$ channels underlying slow IPSCs in rat hippocampal neurons. J. Neurophysiol. 77, 2202.

Diana, M. A., and Marty, A. (2003). Characterization of depolarizationinduced suppression of inhibition using paired interneuron-Purkinje cell recordings. J. Neurosci. 23, 5906-5918.

Dugué, G. P., Dumoulin, A., Triller, A., and Dieudonné, S. (2005).

glycine was loaded into pre-synaptic vesicles in preference to GABA. We thus infer that GABA is not co-released in physiologically relevant amounts at glycinergic synapses onto lumbar motoneurons in juvenile mice.

\section{ACKNOWLEDGMENTS}

We are grateful to Professor H. U. Zeilhofer, from the University of Zurich, for sharing the GlyT2 mice strain. This work was supported by a grant from the Wellcome Trust grant to M. Beato (WT088279AIA). N. J. Moore is funded by a BBSRC Studentship. M. Beato is a Royal Society University Research Fellow.

Target-dependent use of coreleased inhibitory transmitters at central synapses. J. Neurosci. 25, 6490-6498.

Gao, B. X., Stricker, C., and ZiskindConhaim, L. (2001). Transition from GABAergic to glycinergic synaptic transmission in newly formed spinal networks. J. Neurophysiol. 86, 492.

González-Forero, D., and Alvarez, F. J. (2005). Differential postnatal maturation of $\mathrm{GABA}_{\mathrm{A}}$, glycine receptor, and mixed synaptic currents in Renshaw cells and ventral spinal interneurons. J. Neurosci. 25, 2010-2023.

Houston, C. M., Bright, D. P., Sivilotti, L. G., Beato, M., and Smart, T. G. (2009). Intracellular chloride ions regulate the time course of GABAmediated inhibitory synaptic transmission. J. Neurosci. 29, 10416.

Jonas, P., Bischofberger, J., and Sandkühler, J. (1998). Corelease of two fast neurotransmitters at a central synapse. Science 281, 419.

Keller, A. F., Coull, J. A. M., Chéry, N., Poisbeau, P., and De Koninck,
Y. (2001). Region-specific developmental specialization of GABAglycine cosynapses in laminas I-II of the rat spinal dorsal horn. $J$. Neurosci. 21, 7871-7880.

Liu, T. T., Bannatyne, B. A., and Maxwell, D. J. (2010). Organization and neurochemical properties of intersegmental interneurons in the lumbar enlargement of the adult rat. Neuroscience 171, 461-484.

Lu, T., Rubio, M. E., and Trussell, L. O. (2008). Glycinergic transmission shaped by the corelease of GABA in a mammalian auditory synapse. Neuron 57, 524-535.

Ma, W., Behar, T., and Barker, J. L. (1992). Transient expression of GABA immunoreactivity in the developing rat spinal cord. J. Comp. Neurol. 325, 271-290.

Ma, W., Saunders, P. A., Somogyi, R. Poulter, M. O., and Barker, J. L. (1993). Ontogeny of GABAA receptor subunit mRNAs in rat spinal cord and dorsal root ganglia. J. Comp. Neurol. 338, 337-359. 
Mathews, G. C., and Diamond, J. S. (2003). Neuronal glutamate uptake contributes to GABA synthesis and inhibitory synaptic strength. $\mathrm{J}$. Neurosci. 23, 2040.

Moroni, M., Biro, I., Giugliano, M., Vijayan, R., Biggin, P. C., Beato, M., and Sivilotti, L. G. (2011). Chloride ions in the pore of glycine and gaba channels shape the time course and voltage dependence of agonist currents. J. Neurosci. 31, 14095-14106.

O'Brien, J. A., and Berger, A. J. (1999). Cotransmission of GABA and glycine to brain stem motoneurons. J. Neurophysiol. 82, 1638-1641.

Örnung, G., Shupliakov, O., Lindå, H., Ottersen, O. P., Storm-Mathisen, J., Ulfhake, B., and Cullheim, S. (1996). Qualitative and quantitative analysis of glycine-and GABAimmunoreactive nerve terminals on motoneuron cell bodies in the cat spinal cord: a postembedding electron microscopic study.
J. Comp. Neurol. 365, 413-426.

Pitt, S. J., Sivilotti, L. G., and Beato, M. (2008). High intracellular chloride slows the decay of glycinergic currents. J. Neurosci. 28, 11454-11467.

Shimamoto, K., Lebrun, B., YasudaKamatani, Y., Sakaitani, M., Shigeri, Y., Yumoto, N., and Nakajima, T. (1998). DL-Threo$\beta$-benzyloxyaspartate, a potent blocker of excitatory amino acid transporters. Mol. Pharmacol. 53, 195.

Taal, W., and Holstege, J. C. (1994). GABA and glycine frequently colocalize in terminals on cat spinal motoneurons. Neuroreport 5, 2225.

Takahashi, T. (1992). The minimal inhibitory synaptic currents evoked in neonatal rat motoneurones. $J$. Physiol. 450, 593.

Thurbon, D., Lüscher, H. R., Hofstetter, T., and Redman, S. J. (1998). Passive electrical properties of ventral horn neurons in rat spinal cord slices. J. Neurophysiol. 79, 2485-2502.

Todd, A. J., Watt, C., Spike, R. C. and Sieghart, W. (1996). Colocalization of GABA, glycine, and their receptors at synapses in the rat spinal cord. J. Neurosci. 16, 974.

Varoqui, H., Zhu, H., Yao, D., Ming, H., and Erickson, J. D. (2000). Cloning and functional identification of a neuronal glutamine transporter. $J$. Biol. Chem. 275, 4049.

Wojcik, S. M., Katsurabayashi, S., Guillemin, I., Friauf, E., Rosenmund, C., Brose, N., and Rhee, J. S. (2006). A shared vesicular carrier allows synaptic corelease of gaba and glycine. Neuron 50, 575-587.

Zeilhofer, H. U., Studler, B., Arabadzisz, D., Schweizer, C., Ahmadi, S., Layh, B., Bösl, M. R., and Fritschy, J. M. (2005). Glycinergic neurons expressing enhanced green fluorescent protein in bacterial artificial chromosome transgenic mice. J. Comp. Neurol. 482, 123-141.

Conflict of Interest Statement: The authors declare that the research was conducted in the absence of any commercial or financial relationships that could be construed as a potential conflict of interest.

Received: 30 November 2011; accepted: 13 February 2012; published online: 21 March 2012.

Citation: Bhumbra GS, Moore NJ, Moroni $M$ and Beato $M$ (2012) $C o$ release of $G A B A$ does not occur at glycinergic synapses onto lumbar motoneurons in juvenile mice. Front. Cell. Neurosci. 6:8. doi: 10.3389/fncel.2012.00008

Copyright (c) 2012 Bhumbra, Moore, Moroni and Beato. This is an open-access article distributed under the terms of the Creative Commons Attribution Non Commercial License, which permits noncommercial use, distribution, and reproduction in other forums, provided the original authors and source are credited. 